\title{
Optical investigations reveal the effects of 2-aminoethyldiphenyl borate on STIM1 puncta formation
}

\author{
Tao $\mathrm{Yu}^{\dagger}$, Shangbin Chen*, Jingying Pan*, Conglin $\mathrm{Su}^{*}$ and Jun $\mathrm{He}^{*, \S}$ \\ *Division of Histology and Embryology \\ School of Basic Medicine, Tongji Medical College \\ Huazhong University of Science and Technology \\ Wuhan, P. R. China \\ ${ }^{\dagger}$ Wuhan Children's Hospital (Wuhan Maternal and Child Healthcare Hospital) \\ Tongji Medical College, Huazhong University of Science \\ and Technology, Wuhan, P. R. China \\ *Britton Chance Center for Biomedical Photonics \\ Wuhan National Laboratory for Optoelectronics - \\ Huazhong University of Science and Technology, Wuhan, P. R. China \\ \$junhe@mails.tjmu.edu.cn
}

Received 22 May 2017

Accepted 23 July 2017

Published 18 August 2017

\begin{abstract}
2-Aminoethyldiphenyl borate (2-APB) is the most commonly used pharmacological agent in the study of calcium release-activated channels (CRACs); however, its inhibitory mechanism to CRACs remains unclear. To address this issue, we systematically employed confocal imaging, dual-wavelength excitation photometry and FRET to examine the effects of 2-APB on the dynamic activities and function of STIM1 and Orai1, two key components of CRACs. Imaging results support that there are two signaling pathways (Orai1-independent and Orai1-dependent) for the formation of STIM1 puncta. 2-APB could dose dependently block Orai1-independent but not Orai1-dependent STIM1 puncta formation, despite its obvious inhibition effect on storeoperated $\mathrm{Ca}^{2+}$ entry (SOCE). In addition, we found that although 2-APB could not visibly alter near plasma membrane CAD-eYFP localization, it could completely block CAD-YFP-induced constitutive $\mathrm{Ca}^{2+}$ entry and promote the interaction between Orail and CAD by FRET measurements. Therefore, we proposed that inhibitory action of 2-APB on SOCE might attribute to its direct inhibitory effects on Orai1 channel itself, but not the interference on puncta formation between STIM1 and Orai1.
\end{abstract}

Keywords: SOCE; CRAC; 2-aminoethyldiphenyl borate; STIM1; Orai1; confocal imaging; FRET.

$\S_{\text {Corresponding author. }}$

This is an Open Access article published by World Scientific Publishing Company. It is distributed under the terms of the Creative Commons Attribution 4.0 (CC-BY) License. Further distribution of this work is permitted, provided the original work is properly cited. 


\section{Introduction}

In electrically nonexcitable cells, the primary mechanism of $\mathrm{Ca}^{2+}$ influx into cells is a process known as store-operated $\mathrm{Ca}^{2+}$ entry ( $\mathrm{SOCE}$ ), which plays important roles in the control of gene expression, cell growth and differentiation, secretion, $\mathrm{Ca}^{2+}$ homeostasis, and apoptosis. ${ }^{1-3}$ The activation of phospholipase C (PLC)-coupled receptors generates inositol 1,4,5-trisphosphate (IP3) and releases $\mathrm{Ca}^{2+}$ from the lumen of the endoplasmic reticulum (ER). The emptying or decrease of ER luminal $\mathrm{Ca}^{2+}$ in turn triggers the activation of store-operated $\mathrm{Ca}^{2+}$ channels. ${ }^{4,5}$

Over the past decade, mechanisms about activation of store-operated $\mathrm{Ca}^{2+}$ channels (SOCs) by depletion of $\mathrm{Ca}^{2+}$ stores have been explored by numerous laboratories, ${ }^{2}$ and two molecular components of $\mathrm{Ca}^{2+}$-release-activated channel (CRAC) have been found to mediate SOCE after $\mathrm{Ca}^{2+}$ stores depletion: stromal interaction molecule (STIM) molecules (STIM1 and STIM2) that located in the membrane of the $\mathrm{ER}$ as the $\mathrm{Ca}^{2+}$ sensor in the $\mathrm{ER},{ }^{6-11}$ along with Orai proteins (Orai1, 2, and 3) that located in the plasma membrane as the poreforming subunits of CRAC. ${ }^{12-14}$ It is now evident that $\mathrm{Ca}^{2+}$ stores depletion causes dissociation of $\mathrm{Ca}^{2+}$ from the EF-hand domain of the N-terminus (NT) in STIM proteins. That makes the unfolding of the STIM1-NT and the oligomerization of neighboring STIM molecules, triggering STIM1 translocation to form clusters in junctional ER sites, which locate in close proximity to the plasma membrane and are commonly referred to as "puncta" ${ }^{6,15-17}$; whereas Orai1 accumulates in the areas of plasma membrane apposed STIM1 puncta. $8,9,18$ STIM1 interacts directly with the C- and N-termini of ORAI1 to activate CRACs, ${ }^{15,19-24}$ and several interacting partners within the STIM1-Orai1 complex indirectly regulate the activity of CRACs. ${ }^{24-27}$ Knowledge of the key molecular components of the CRAC has allowed investigation of the cellular and molecular mechanisms of pharmacological modulators.

2-Aminoethoxydiphenyl borate (2-APB) is a popular pharmacological agent in the study of CRAC/store-operated channels. This drug was originally introduced as a membrane-permeant inhibitor of the inositol 1,4,5-trisphosphate receptor. ${ }^{28}$ Although it has subsequently been found to affect a variety of ion channels and transport processes, the most reliable and best-studied effect of 2-APB is its ability to affect the activity of CRAC. ${ }^{3,29}$ Its effects are bimodal, further potentiating CRAC currents at low concentrations $(1-10 \mu \mathrm{M})$, but transiently enhancing and then completely inhibiting CRAC currents at high concentrations $(>20 \mu \mathrm{M}) \cdot{ }^{30-32}$ 2-APB also reduces $\mathrm{Ca}^{2+}$-dependent fast inactivation of CRAC in parallel with the slow development of its inhibitory effects at high concentrations. ${ }^{32}$ Recently, it has been reported that both STIM proteins (STIM1 and STIM2) and all three CRACs (Orai1, Orai2, and Orai3) can interact with each other and that 2-APB has differential effects on the three CRAC subtypes. $^{30,33,34}$ The mechanism of 2 -APB action on CRAC still remains unclear, but the complex effects elicited by 2-APB suggest that this compound may target multiple processes of CRAC activation. Discoveries of Orai1 and STIM1 have prompted direct experiments to explore the molecular mechanism of 2-APB modulation for better understanding of the mechanisms by which CRACs are regulated.

Benefited from the advantages of fluorescence labeling and confocal microscope, confocal imaging enables us to visualize the dynamic movement of STIM1 and Orail and to investigate the effect of 2-APB on the dynamic movement of STIM1 and Orai1. Since FRET is a sensitive distancedependent proximity probe, allowing the detection of donor-acceptor interactions on a molecular scale (1-10 nm), we also have employed FRET to monitor the association of STIM1 or its mutant segments with Orai1 and subsequent molecular rearrangements in the CRAC. We examined the protein targets of channel regulation by 2 -APB. We found that 2-APB has different effects on two mechanisms of STIM1 puncta formation and proposed that inhibitory action of 2-APB on SOCE might attribute to its direct inhibitory effects on Orail channel itself, but not the interference on puncta formation between STIM1 and Orai1.

\section{Methods}

\subsection{Cell culture and transfection}

HEK293T cells (ATCC) were cultured in Dulbecco's modified Eagle's medium (DMEM) containing $10 \%$ heat-inactivated fetal bovine serum, $50 \mathrm{U} / \mathrm{mL}$ penicillin, and $50 \mathrm{mg} / \mathrm{mL}$ streptomycin. Cells were maintained at $37^{\circ} \mathrm{C}$ in a humidified 
incubator set at $5 \% \mathrm{CO}_{2}$ and were plated onto $30 \mathrm{~mm}$ round glass coverslips in a six-well plate. On the following day, different plasmids mentioned below were transfected into HEK293T cells by means of Lipofectamine 2000 (Invitrogen) as per manufacturer's instruction. Six hours later the Opti-MEM medium bathing the cells was replaced with complete DMEM and maintained in culture overnight. After $48 \mathrm{~h}$, cells were taken out for detection.

\subsection{Plasmids construction}

To study the function of different domains of STIM1 in the formation of STIM1 puncta induced by $\mathrm{Ca}^{2+}$ store depletion, we constructed different truncated forms of STIM1 and expressed alone or co-expressed them with wild-type Orai1 in HEK293 cells. The N-terminus tagged mCherry-STIM1 construct and GFP-PLC $\delta$-PH were the kind gifts of Dr Richard S. Lewis (Stanford University, America) and Dr Tamas Balla (National Institutes of Health, America). Full-length human Orai1 was amplified by using PCR method from human placenta cDNA library (Invitrogen), which was, respectively, cloned into pEYFP-N1, pECFP-N1, and mKate-N1 expression vectors (Clontech). STIM1- $\Delta \mathrm{K}$ (672-685) was amplified by PCR from mCherry-STIM1 and ligated into a pcDNA3.1 $\mathrm{Zeo}(-)$ vector; this construct was devoid of the C-terminal polybasic domain (aa 672-685) of STIM1. Amplification products of CAD were cloned into the pEYFP-N1 expression vector to yield CAD-EYFP construct, which only includes the CRAC activation domain (CAD, aa 342-448). The K-rich region (aa 670-685) of STIM1 and STIM2 (aa 728-746) was amplified and ligated into pEGFP-N1 vector to yield GFP$\mathrm{K}^{\text {STIM1 }}$ and GFP-K ${ }^{\text {STIM2 }}$ constructs, respectively. C-terminal polybasic clusters (aa 194-219) of Rit (PM-localized small GTPase) were amplified and inserted into pEGFP-N1 vector to yield GFP-Rit tail construct. All constructs were verified by sequencing.

\subsection{Solutions and chemicals}

In confocal imaging experiments, we used standard extracellular Ringer's solution containing the following (in $\mathrm{mM}$ ): $150 \mathrm{NaCl}, 5 \mathrm{KCl}, 1.8 \mathrm{CaCl}_{2}, 1 \mathrm{MgCl}_{2}$, 8 glucose, and 10 HEPES (pH 7.4, adjusted with
$\mathrm{NaOH}$ ). The $\mathrm{CaCl}_{2}$ was replaced by $1 \mathrm{mM}$ EGTA and $2 \mathrm{mM} \mathrm{MgCl}_{2}$ in $\mathrm{Ca}^{2+}$-free Ringer's solution. Stock solutions of thapsigargin (TG) and 2-APB were prepared in Me2SO at a concentration of $1 \mathrm{mM}$. fura$2 / \mathrm{AM}$ was purchased from Invitrogen. Unless otherwise specified, all reagents and chemicals were from Sigma-Aldrich.

\subsection{Confocal microscopy imaging}

Experiments were performed using the Olympus FV500 laser scanning confocal microscopy system (Olympus, Tokyo). Cover slips containing cells were placed into a perfusion chamber on a stage of an inverted Olympus IX70 microscope. Structural data acquisition was performed in the sequential line mode for the best spatiotemporal reliability. Live cells were examined in a confocal microscope at the cell footprint to better visualize near PM STIM1 puncta. The sample area is scanned at resolution of $512 \times 512$ pixels. eGFP, eYFP, and mCherry (mKate) were excited at 488,514 , and $543 \mathrm{~nm}$, respectively. All experiments were performed at $22-25^{\circ} \mathrm{C}$.

For FRET experiments, the donor dye eCFP was excited using the $405 \mathrm{~nm}$ diode laser. Donor and acceptor dye fluorescence signals were recorded in line mode simultaneously in two independent channels set to detect light in the ranges $450-510 \mathrm{~nm}$ and 540-625 nm, respectively. FRET was calculated as the ratio of eYFP (FRET) to eCFP $\left(\mathrm{I}_{\mathrm{DD}}\right)$ intensity. The images were off-line analyzed using Fluoview Imaging Software (Olympus, Tokyo).

\subsection{Intracellular $\mathrm{Ca}^{2+}$ measurements}

Cells were loaded with $2 \mu \mathrm{M}$ fura- $2 / \mathrm{AM}$ at $37^{\circ} \mathrm{C}$ for $30 \mathrm{~min}$ in standard Ringer's solution. $\left[\mathrm{Ca}^{2+}\right]_{i}$ was measured using a dual-wavelength excitation (340/ $380 \mathrm{~nm}$ ) photometry system on an inverted microscope (TE2000, Nikon, Japan) equipped with a polychromatic xenon light source (TILL Photonics, Germany). The emission was collected at $510 \mathrm{~nm}$ with a photodiode controlled by the TILL photometry system and X-Chart extension of Pulse software (HEKA, Lambrecht, Germany). $\mathrm{Ca}^{2+}$ fluctuations are reported as the ratio of fluorescence emission at the two excitation wavelengths. Cells co-transfected with mCherry-STIM1- $\Delta \mathrm{K}$ and Orai1-eYFP STIM1 were chosen based on their fluorescence when excited at $514 \mathrm{~nm}$. 


\section{Results}

\subsection{Confirmation of Orai1-independent and Orai1-dependent STIM1 rearranges to ER-PM junctions}

In cells transfected with mCherry-labeled STIM1 alone, over-expressed STIM1 was observed to form distinct puncta after depletion of $\mathrm{Ca}^{2+}$ stores with TG [Fig. 1(a)]. In contrast, clustering of Orai1 was not observed in cells overexpressed Orai1-eYFP alone after store depletion [TG; Fig. 1(b)]. However, when expressed together, mCherry-STIM1 and
Orai1-eYFP form colocalized puncta after store depletion [Fig. 1(c)]. mCh-STIM1- $\Delta \mathrm{K}$ failed to form puncta after store depletion when expressed alone in HEK293 cells [Fig. 1(d)]; but when co-expressed with Orai1-eYFP, both proteins restored their ability to form colocalized puncta after $\mathrm{Ca}^{2+}$ store depletion [Fig. 1(e)].

As shown in Fig. 1(f), when expressed alone in cells, CAD-eYFP was localized diffusely throughout the cytoplasm, but co-expression with Orai1-mKate led to a dramatic recruitment of CAD-eYFP to the plasma membrane, suggesting that the two proteins

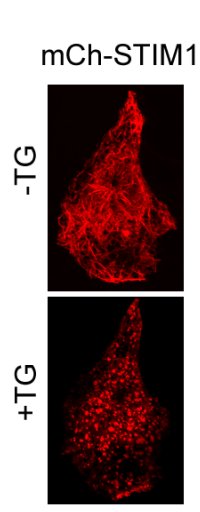

(a)

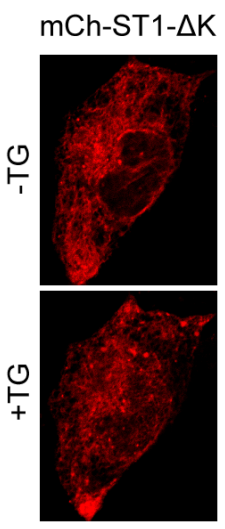

(d)

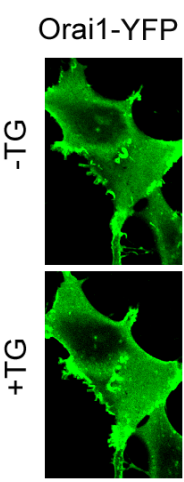

(b)
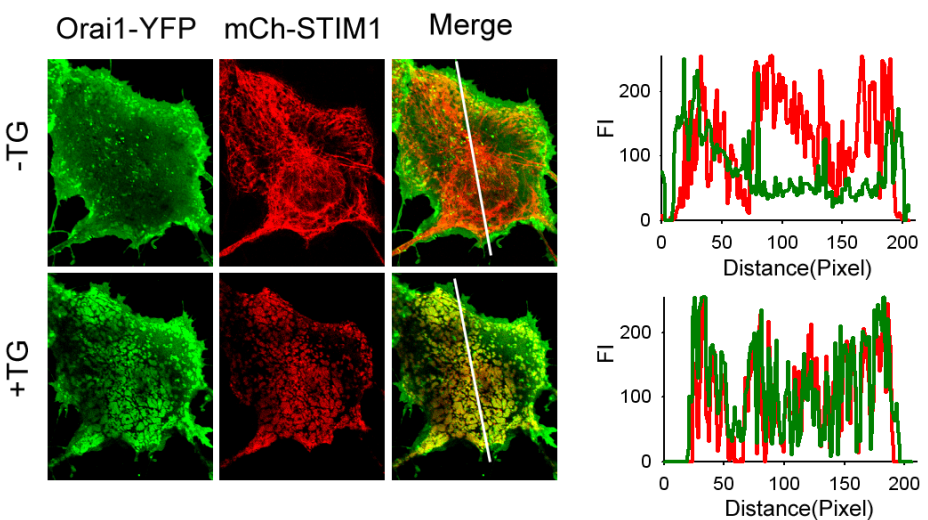

(c)
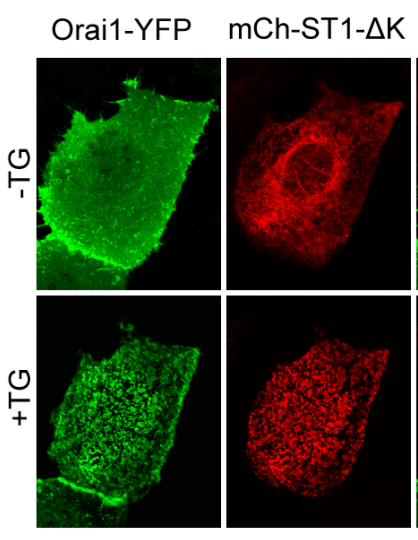

(e)

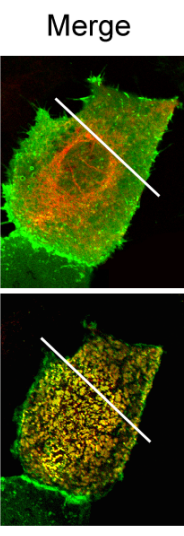

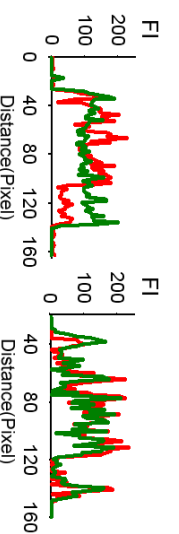

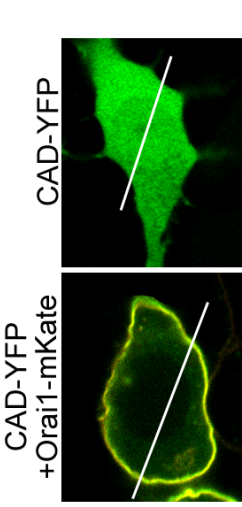

(f)

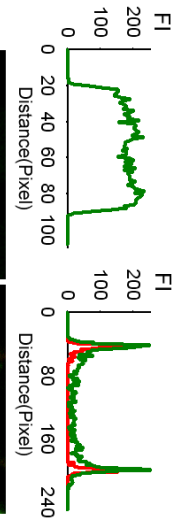

Fig. 1. STIM1 recruits to ER-PM junctions to form puncta through Orai1-dependent and -independent mechanisms. (a) Depletion of the ER $\mathrm{Ca}^{2+}$ store with TG induces mCherry-STIM1 expressed alone in HEK293 cells aggregation into puncta underneath the plasma membrane. After depletion, puncta are visible at the cell footprint. (b) Store depletion fails to evoke clustering of Orai1-eYFP when overexpressed alone as observed under confocal microscope in HEK293 cells. (c) When expressed together, mCherry-STIM1 and Orail-eYFP form colocalized puncta after store depletion. The right panels show spatial profiles of fluorescence intensity (FI) of Orai1-eYFP (green) and mCherry-STIM1 (red) along the lines imposed on the images. (d) mCherry-STIM1$\Delta \mathrm{K}$ expressed alone in HEK293 cells fails to form puncta after store depletion. (e) When expressed together, mCherry-STIM1- $\Delta \mathrm{K}$ and Orai1-eYFP form colocalized puncta after store depletion. (f) CAD-eYFP is diffuse cytoplasm distribution when expressed alone in a HEK293 cell (top), but the co-expression with Orai1 led to a dramatic recruitment of CAD-eYFP to the cell periphery (bottom). The right panels show spatial profiles of fluorescence intensity of CAD-eYFP (green) and Orai1-mKate (red) along the lines imposed on the images. 
form a complex. Our FRET experiments also demonstrated that there is direct interaction between CAD domain and Orai1 (data not shown). In summary, our results confirmed that the polybasic domain and CAD domain were necessary for the recruitment of STIM1 to ER-PM junctions. STIM1 can rearrange to ER-PM junctions in Orai1independent and Orai1-dependent mechanism, whereas Orai1 recruitment to these sites depends on binding to CAD domain of STIM1.

\subsection{2-APB blocks the Orai1-independent STIM1 puncta formation}

2-APB elicits dual effects, potentiating CRAC currents at low concentrations $(1-10 \mu \mathrm{M})$, but completely blocking SOC activity at high concentrations $(>20 \mu \mathrm{M})$. Our data also confirmed that 2-APB was a reliable blocker of store-operated $\mathrm{Ca}^{2+}$ entry (data not shown). Thus, we considered whether 2-APB inhibits SOCE by blocking the rearrangement of STIM1 that occurs when $\mathrm{Ca}^{2+}$ stores are depleted. To test this, we imaged eYFP-STIM1-expressing
HEK293 cells by confocal microscopy at the cell footprint. As shown in Fig. 2(a), store depletion with TG caused rearrangement of EYFP-STIM1 from tubular structures into discrete puncta underneath the plasma membrane. After the addition of $50 \mu \mathrm{M}$ 2-APB, the punctate structures were completely dispersed, and eYFP-STIM1 returned to tubular structures; pretreatment of cells with $50 \mu \mathrm{M} 2-\mathrm{APB}$ could also completely prevent eYFP-STIM1 from migrating to near membrane regions to form puncta in response to store depletion [Fig. 2(b)].

To more quantitatively assess the effect of 2-APB on STIM1 relocation, time-lapse confocal imaging at the cell footprint was performed on eYFP-STIM1expressing cells treated with various concentrations of 2-APB. As seen in the left trace in Fig. 2(d), the fluorescence intensity measured by confocal microscopy increased largely following store depletion with TG. In contrast, cells treated with higher concentrations of 2-APB $(>25 \mu \mathrm{M})$ for 5 min before store depletion exhibited little to no increase in confocal fluorescence intensity following store depletion. However, lower concentrations $(<20 \mu \mathrm{M})$ of 2 -APB
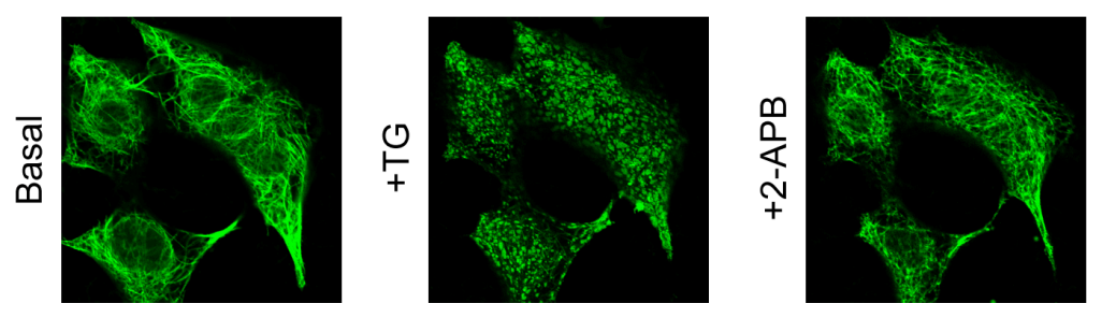

(a)
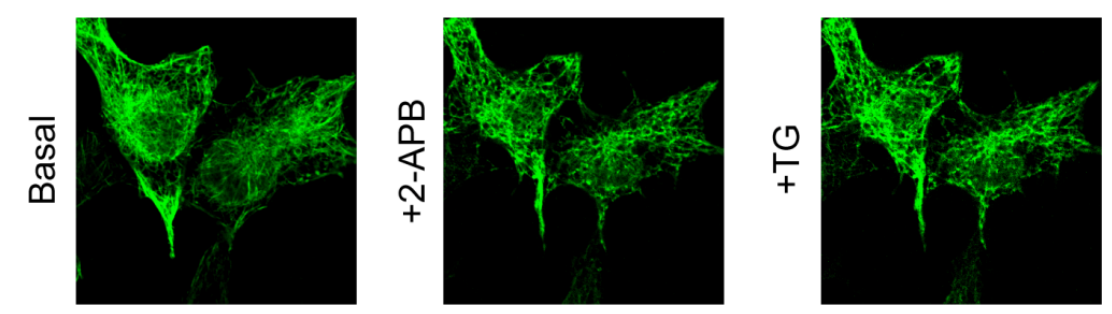

(b)

Fig. 2. 2-APB dose-dependently inhibits or reverses rearrangement of STIM1. (a) $50 \mu \mathrm{M}$ 2-APB reverses near plasma membrane punctate STIM1 localization in HEK293 cells. eYFP-STIM1-expressing HEK293 cells were examined in a confocal microscope at the cell footprint with replete $\mathrm{Ca}^{2+}$ stores (left) following store depletion with $2 \mu \mathrm{M}$ TG (middle) and 5 min following treatment with $50 \mu \mathrm{M} 2$-APB (right). (b) $50 \mu \mathrm{M}$ 2-APB inhibits the formation of STIM1 puncta in near plasma membrane. Confocal imaging was carried out in experiments in which cells expressing eYFP-STIM1 were pretreated with $50 \mu \mathrm{M} 2$-APB for 5 min prior to store depletion with TG. (c) Time-lapse confocal imaging at the cell footprint was performed on eYFP-STIM1-expressing cells. Shown are fluorescence intensity ratio $(F / F 0)$ profiles for $0,25,50$, and $100 \mu \mathrm{M} 2$-APB-treated cells. As indicated, cells were treated with $2 \mu \mathrm{M}$ TG followed $5 \mathrm{~min}$ later by application of various concentration 2-APB in the $0 \mathrm{mM}$ extracellular $\mathrm{Ca}^{2+}$ bath solution. (d) Confocal imaging was performed on live cells as described in (c), but 2-APB was added before store depletion with TG. (e) Left and right bar graphs show the change of confocal fluorescence intensity ratio from various concentration 2-APB-treated experiments shown in (c) and (d), respectively. 

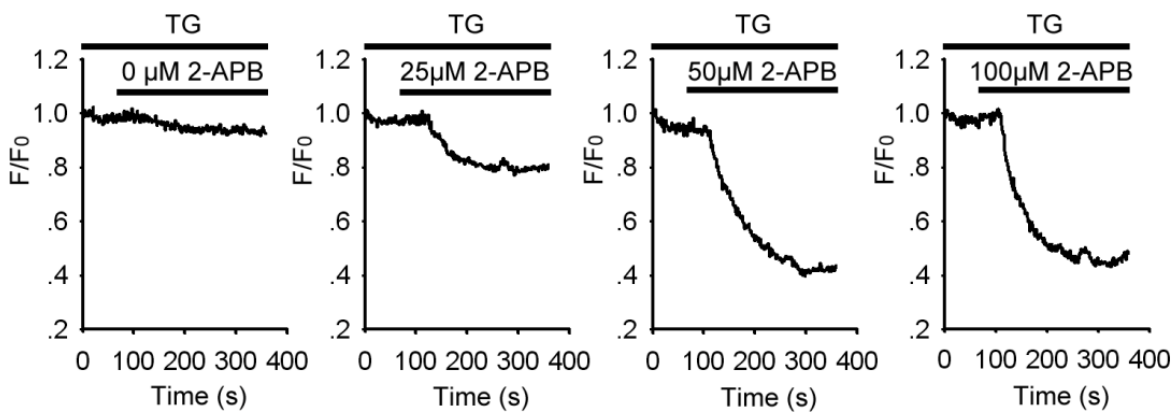

(c)
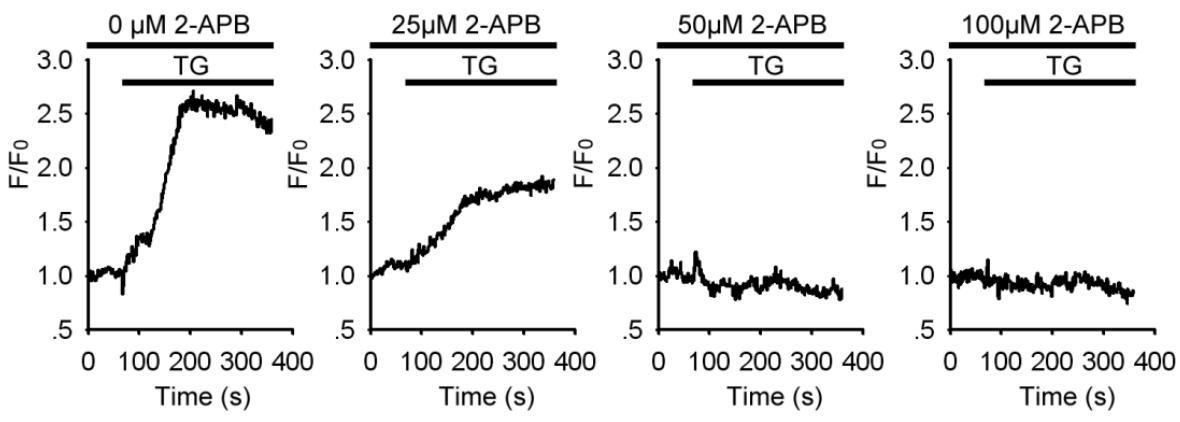

(d)
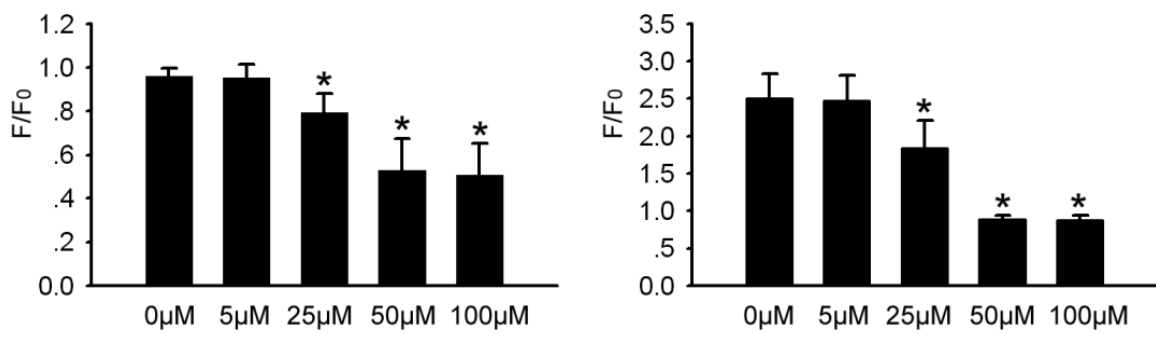

(e)

Fig. 2. (Continued)

had no effect on the enhancement of fluorescence intensity induced by TG [Fig. 2(e)]. Furthermore, as shown in Figs. 2(c) and 2(e), eYFP-STIM1expressing cells were pretreated with TG for $5 \mathrm{~min}$, and 2-APB was then added in the continued presence of TG. After the addition of 2-APB, eYFP-STIM1 near membrane fluorescence intensity decreased dose-dependently.

\subsection{2-APB cannot dissociate the interaction between polybasic domain and PM phosphoinositides}

It has been suggested that the C-terminal polybasic domain of STIM1 is an electrostatic phosphoinositide lipid-binding motif, which directs Orai1-independent formation of STIM1 puncta. We hypothesized that 2-APB inhibits or reverses rearrangement of STIM1 by disturbing the electrostatic attraction between STIM1 polybasic domain and PM phosphoinositides.

To test this, we constructed plasmids of GFP$\mathrm{K}^{\text {STIM1 }}$ (contained the polybasic domains of STIM1) and GFP-K ${ }^{\text {STIM2 }}$ (contained the polybasic domains of STIM2) to monitor the intracellular localization of isolated STIM1/2 polybasic domain by confocal images in live cells. As shown in Fig. 3(a), GFP-K ${ }^{\text {STIM1 }}$ was uniformly distributed throughout the cell body and processes of HEK293 cells. GFP$\mathrm{K}^{\text {STIM2 }}$ was also uniformly distributed throughout the cell body; however, it partially localized to the PM in the processes of cells [Fig. 3(b)]. Figures 3(c) and $3(\mathrm{~g})$ showed that $50 \mu \mathrm{M}$ 2-APB completely 

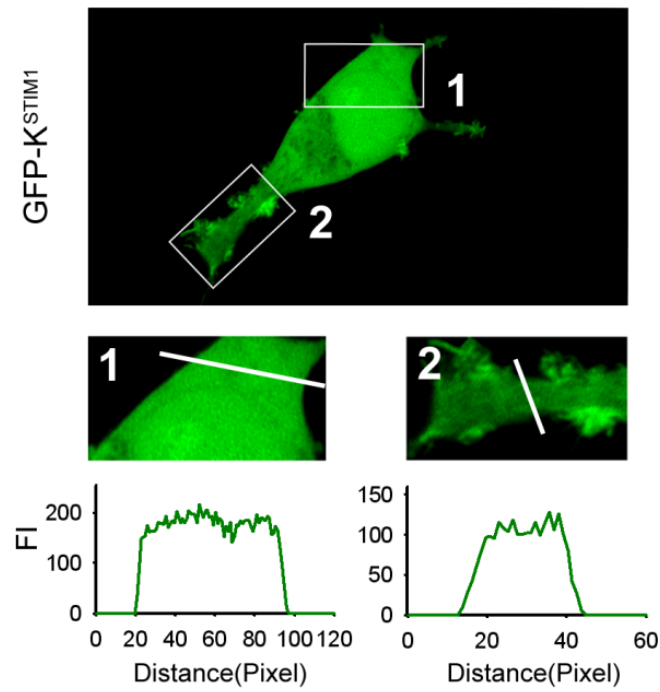

(a)

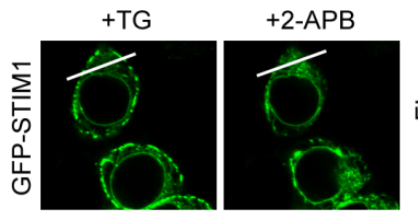

(c)

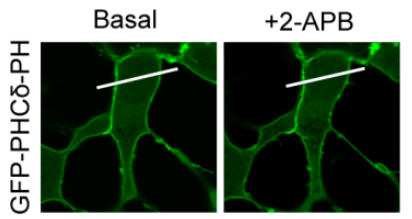

(e)
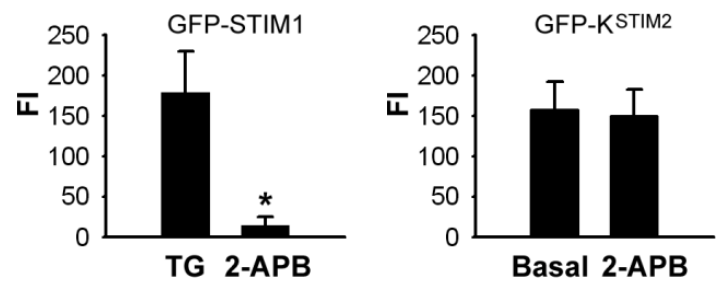

(g)
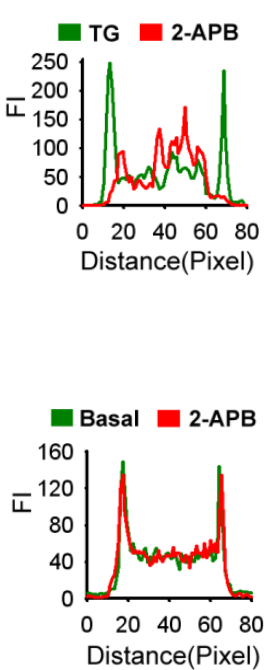

(h)
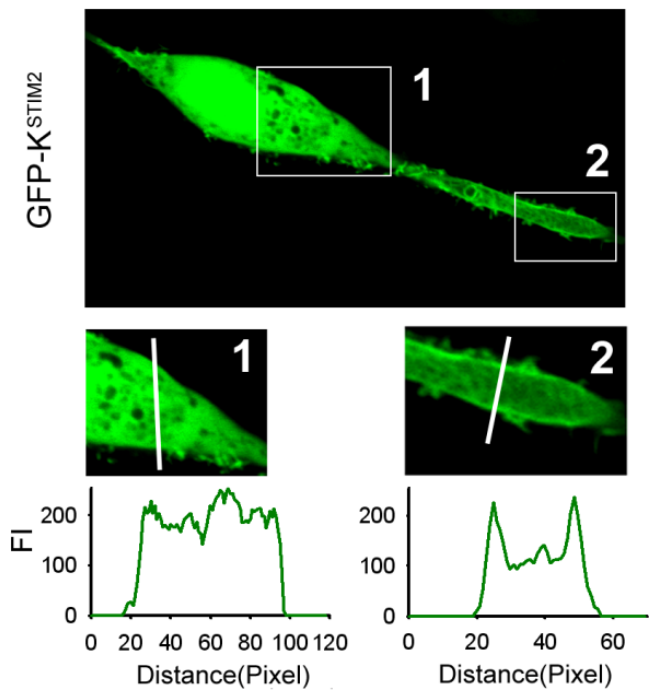

(b)
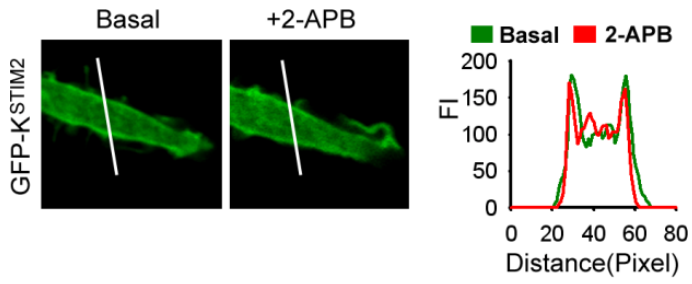

(d)
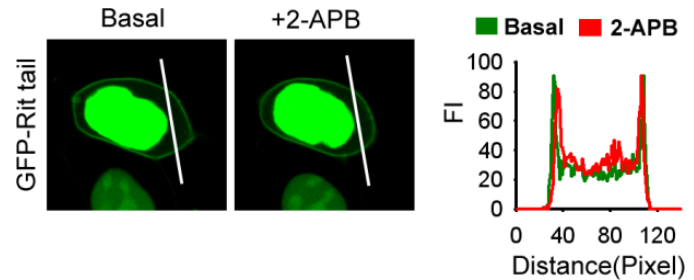

(f)

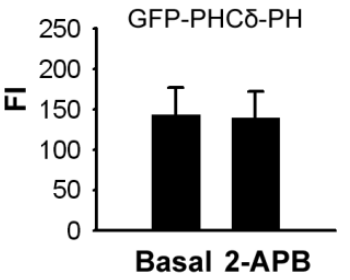

(i)

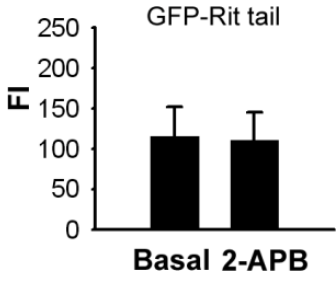

(j)

Fig. 3. 2-APB has no effect on the binding between polybasic domain and PM phosphoinositides. (a) Representative confocal

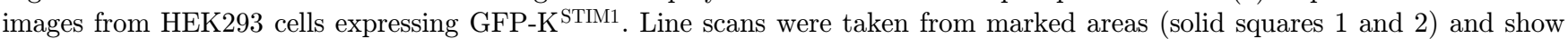
profiles of fluorescence intensity against line distance. (b) Representative confocal images from HEK293 cells expressing GFP-K $^{\text {STIM2 }}$. Line scans were taken from marked areas (1 and 2). Line scans from areas 2 show that GFP-K ${ }^{\text {STIM2 }}$ is partially localized to the PM. (c) Confocal images of GFP-STIM1-expressing cells in the presence of TG (left panel) and 5 min following addition of $50 \mu \mathrm{M} 2$-APB in the continued presence of TG (center panel). Right panel shows merged profiles of fluorescence intensity against line distance. $(\mathrm{d}-\mathrm{f})$ Representative confocal images from HEK293 cells expressing GFP-K ${ }^{\mathrm{STIM} 2}$, GFP-PLC $\delta$-PH, and GFPRit tail, respectively, in the absence (left panel) and presence (center panel) of $50 \mu \mathrm{M} 2-\mathrm{APB}$. (g-j) Bar graphs show the change of $\mathrm{PM}$ fluorescence intensities from 2-APB-treated experiments shown in (c)-(f), respectively. 
dispersed near plasma membrane STIM1 puncta in HEK293 cells. Strikingly, we found that 2-APB had no effect on the near plasma membrane GFP$\mathrm{K}^{\text {STIM2 }}$ localization in the processes of HEK293 cells [Figs. 3(d) and 3(h)]. We also found that 2-APB could not disperse the PM localization of GFP-Rit-tail and GFP-PLC $\delta$-PH [Figs. 3(e), 3(f), 3(i), and 3(j)].

\subsection{Effects of 2-APB on STIM1-Orai1 associations}

Figure 4(a) shows confocal imaging experiments, in which $50 \mu \mathrm{M}$ 2-APB much less effectively (compared with eYFP-STIM1 alone; see Fig. 2) dispersed store depletion-dependent colocalized puncta of STIM1 and Orai1 in HEK293 cells co-expressing mCherrySTIM1 with Orai1-eYFP. Neither lower $(5-20 \mu \mathrm{M})$ nor higher $(100 \mu \mathrm{M})$ concentrations of 2-APB visibly altered TG-induced STIM1 and Orai1 puncta in HEK293 cells co-expressing STIM1 with Orai1 (data not shown). In addition, unlike for cells expressing eYFP-STIM1 alone, cells co-expressing mCherrySTIM1 with Orai1-eYFP (pretreated with $50 \mu \mathrm{M}$ 2-APB for $5 \mathrm{~min}$ ) showed significant formation of colocalized puncta after TG treatment [Fig. 4(b)]. Higher concentrations of 2-APB (100-200 $\mu \mathrm{M})$ also
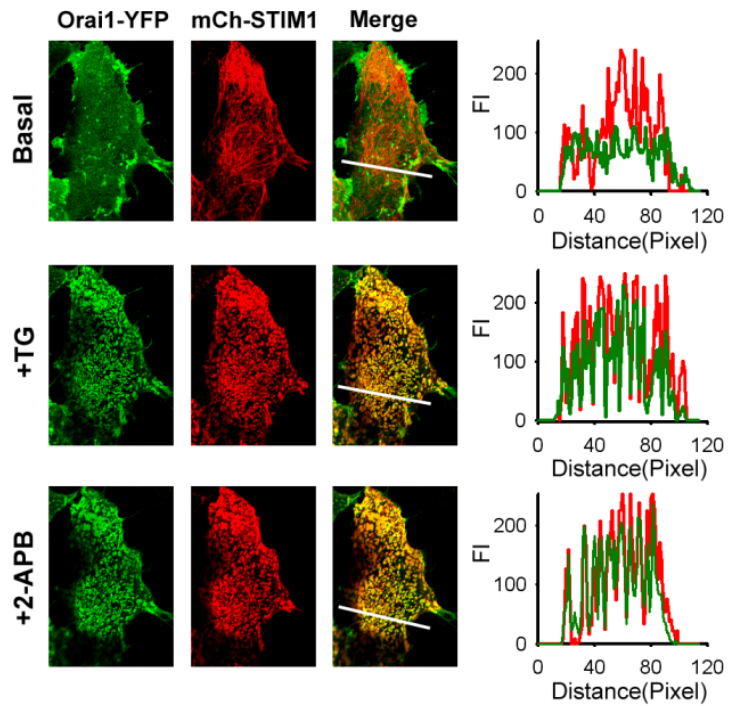

(a) failed to inhibit the TG-induced formation of STIM1 and Orail colocalized puncta (data not shown).

\subsection{2-APB failed to block the Orai1-dependent STIM1 puncta formation}

Figure 5 (a) shows that $50 \mu \mathrm{M} 2$-APB failed to disperse the colocalized puncta of mCherry-STIM1- $\Delta \mathrm{K}$ and Orai1-eYFP STIM1 in store-depleted cells. Neither lower $(5-20 \mu \mathrm{M})$ nor higher $(100 \mu \mathrm{M})$ concentrations of $2-\mathrm{APB}$ visibly altered STIM1- $\Delta \mathrm{K}$ and Orai1 puncta in store-depleted cells (data not shown). However, 2-APB nearly completely blocked SOCE in HEK293 cells expressing mCherrySTIM1- $\Delta \mathrm{K}$ with Orai1-eYFP [Fig. 5(c)]. Our data also demonstrated that SOCE in HEK293 cells expressing mCherry-STIM1- $\Delta \mathrm{K}$ with Orai1-eYFP was much larger than the SOCE in wild-type HEK293 cells [Figs. 5(c)-5(e)]. Additionally, similar to cells co-expressing mCherry-STIM1 with Orai1eYFP, pretreatment of $50 \mu \mathrm{M} 2-\mathrm{APB}$ prior to store depletion did not diminish the ability of TG to subsequently induce puncta formation [Fig. 5(b)]. Higher concentrations of 2-APB $(100-200 \mu \mathrm{M})$ still failed to inhibit the TG-induced formation of
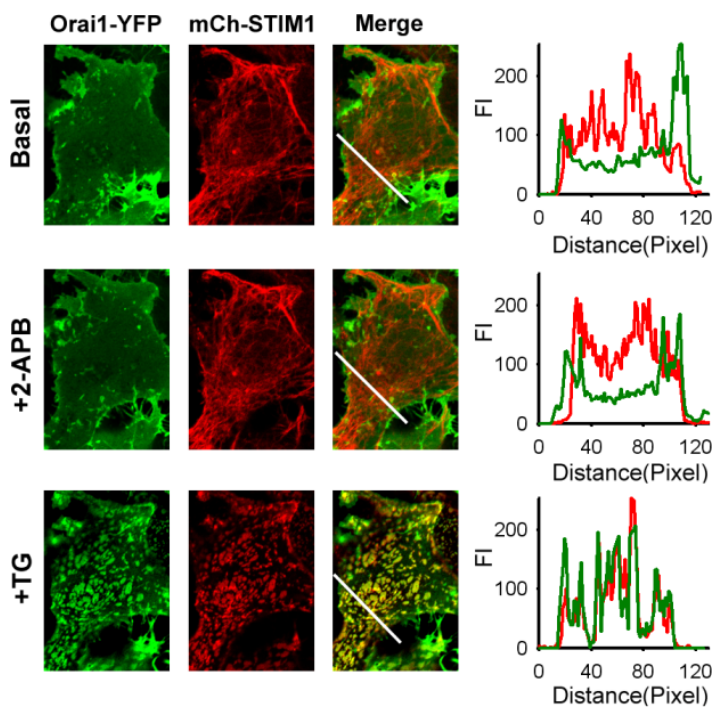

(b)

Fig. 4. 2-APB cannot reverse or prevent STIM1 localization in near plasma membrane puncta in HEK293 cells expressing mCherry-STIM1 and Orai1-eYFP. (a) After $\mathrm{Ca}^{2+}$ store depletion, mCherry-STIM1 and Orai1-eYFP formed colocalized puncta (+TG); $50 \mu \mathrm{M}$ 2-APB failed to disperse these colocalized puncta in HEK293 cells (+2-APB). The right panels show spatial profiles of fluorescence intensity of Orai1-eYFP (green) and mCherry-STIM1 (red) along the lines imposed on the images. (b) Cells coexpressing mCherry-STIM1 with Orai1-eYFP and pretreated with $50 \mu \mathrm{M}$ 2-APB for 5 min showed significant formation of colocalized puncta after TG treatment. 


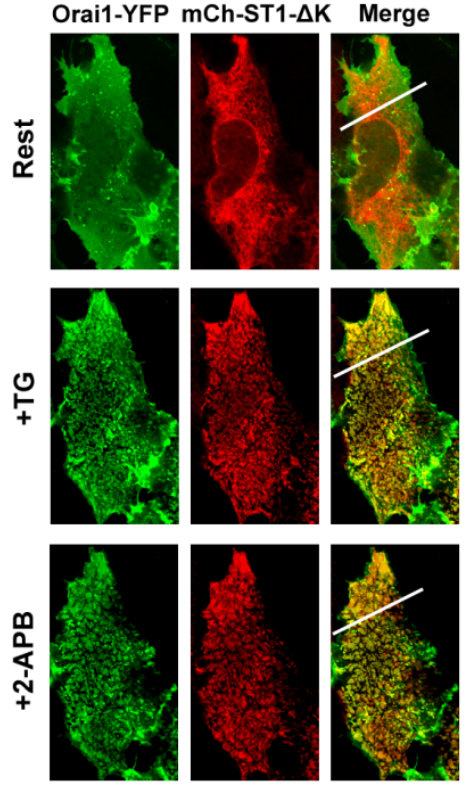

(a)

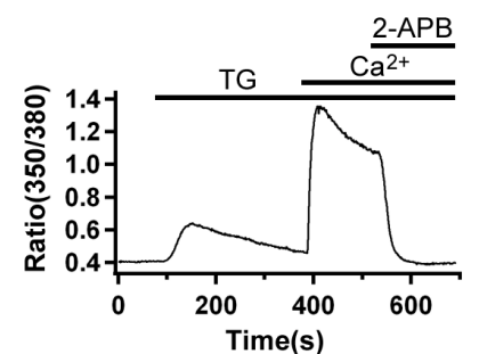

(c)
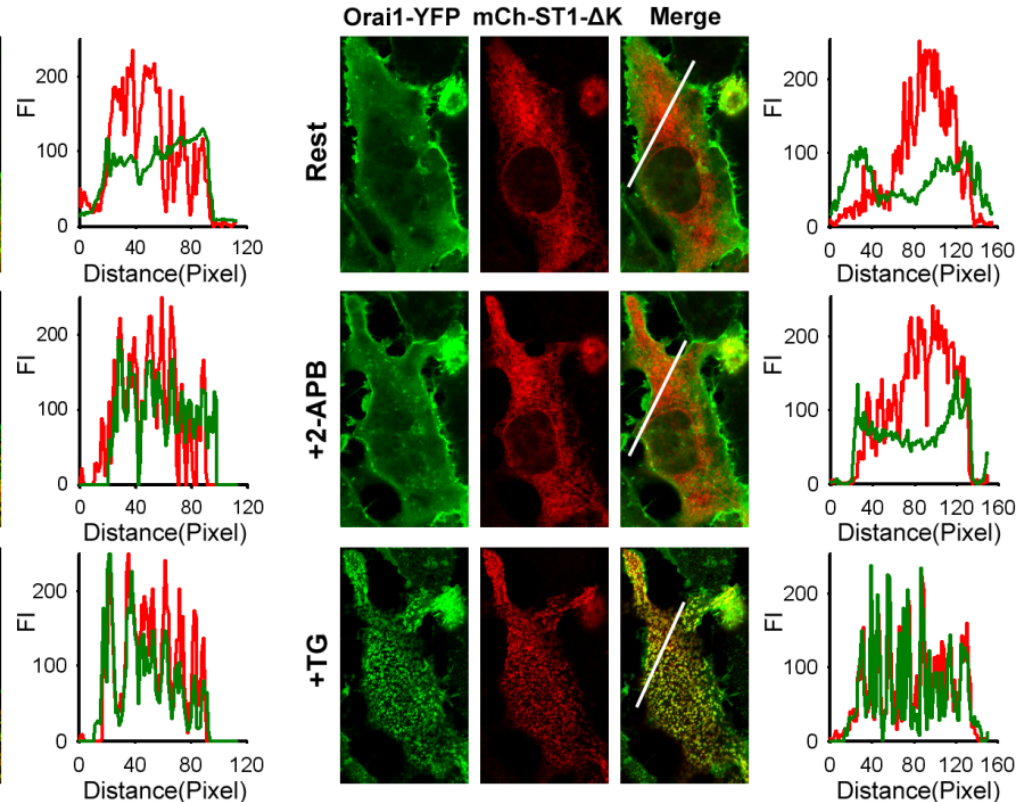

(b)

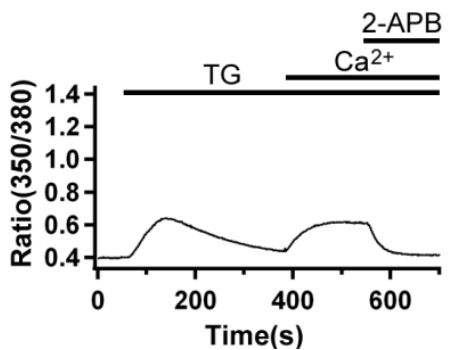

(d)

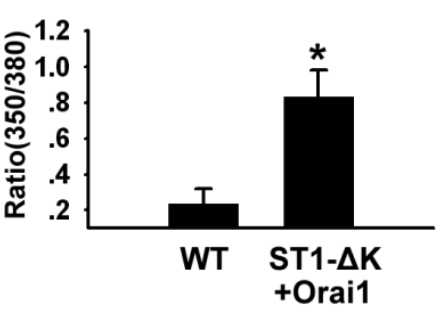

(e)

Fig. 5. 2-APB failed to reverse or prevent STIM1- $\Delta \mathrm{K}$ localization in near plasma membrane puncta in HEK293 cells co-expressing mCherry-STIM1- $\triangle \mathrm{K}$ and Orai1-eYFP. (a) After $\mathrm{Ca}^{2+}$ store depletion, mCherry-STIM1- $\Delta \mathrm{K}$ co-accumulates with Orai1 in puncta (middle); $50 \mu \mathrm{M}$ 2-APB failed to reverse STIM1- $\Delta \mathrm{K}$ localization in near plasma membrane puncta in HEK293T cells (bottom). (b) Confocal imaging was carried out on cells expressing mCherry-STIM1- $\Delta \mathrm{K}$ with Orai1-eYFP, in which $50 \mu \mathrm{M} 2$-APB was added 5 min prior to store depletion with TG. After pretreatment of cells with $50 \mu \mathrm{M} 2$-APB, mCherry-STIM1- $\Delta \mathrm{K}$ redistributed into puncta and clustered Orai1 after depletion. (c) SOCE was measured in HEK293 cells co-expressing STIM1- $\Delta \mathrm{K}$ and Orai1 using TG $(2 \mu \mathrm{M})$. TG was applied under $\mathrm{Ca}^{2+}$-free conditions for $5 \mathrm{~min}$ to deplete ER $\mathrm{Ca}^{2+}$ stores followed by addback of $1.8 \mathrm{mM} \mathrm{Ca}{ }^{2+}$ to assess SOCE. Traces indicate the changes in the ratio of Fura-2 fluorescence intensities over time in selected cells. (d) SOCE was measured in wild-type HEK293 cells in the same experimental paradigm as (c). (e) Averaged peak Ca ${ }^{2+}$ elevation by re-addition of $1.8 \mathrm{mM} \mathrm{Ca}^{2+}$ after store depletion from experiments carried out as described in (c) $(n=22)$ and $(\mathrm{d})(n=23)$. Significances of the $t$-test are labeled on the histogram.

puncta in HEK293 cells co-expressing STIM1- $\Delta \mathrm{K}$ with Orai1 (data not shown).

\subsection{2-APB increased the interaction between CAD-YFP and Orai1-CFP}

When co-expressed with Orai1-mKate, CAD-eYFP was recruited to the plasma membrane and colocalized with Orai1-mKate [Fig. 6(a), top]. An aliquot of $50 \mu \mathrm{M} 2-\mathrm{APB}$ had no effect on the near plasma membrane CAD-eYFP localization in HEK293 cells [Figs. 6(a) and 6(d), bottom]. Neither lower $(5-25 \mu \mathrm{M})$ nor higher $(100 \mu \mathrm{M})$ concentrations of 2-APB visibly altered near plasma membrane CAD-eYFP localization [Figs. 6(b), 6(c), and 6(e)]. However, in HEK293 cells co-expressing CAD-eYFP and Orai1-eCFP, CAD-eYFP caused robust constitutive $\mathrm{Ca}^{2+}$ entry rapidly blocked by 50 IM 2-APB [Figs. 6(f) and 6(g)]. Furthermore, we utilized FRET technique to demonstrate that high 

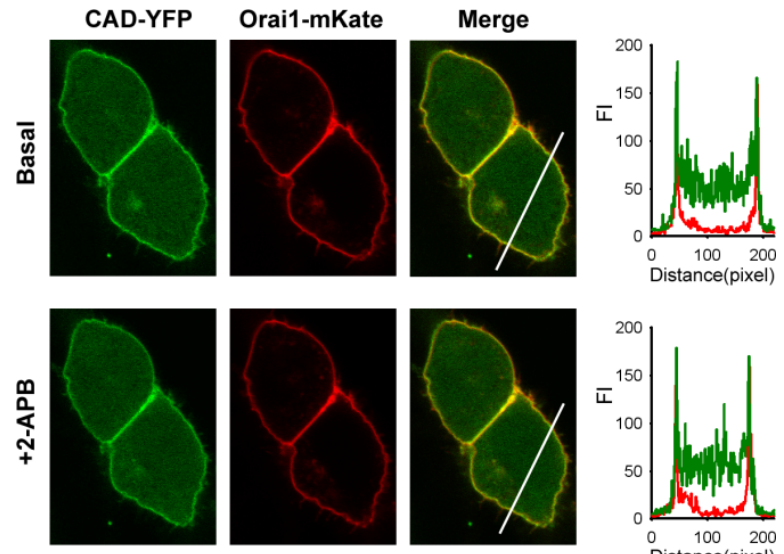
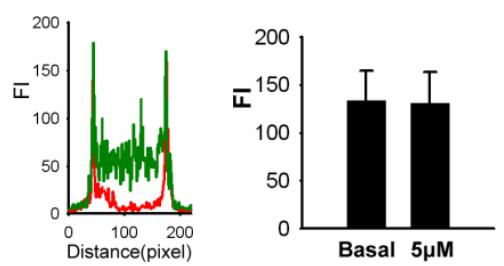

(b)

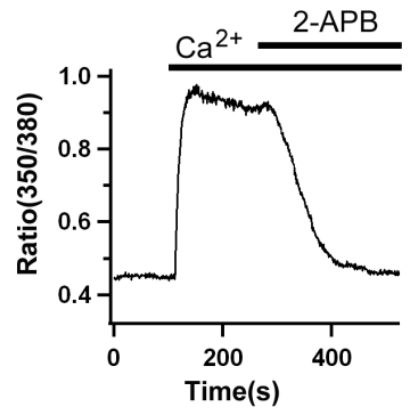

(f)

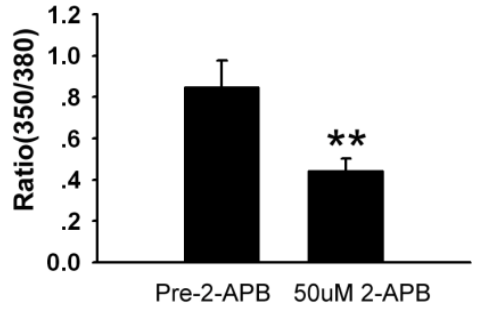

(g)

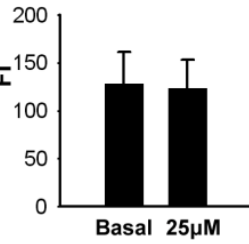

(c)

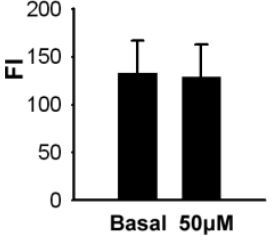

(d)

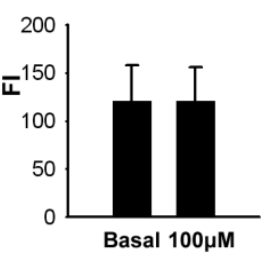

(e)

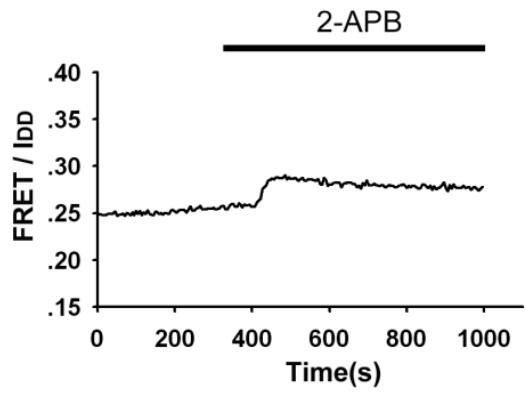

(h)

Fig. 6. 2-APB could not visibly alter near PM CAD-eYFP localization, but enhance the interaction between Orai1 and CAD. (a) Co-expression with Orai1-mKate led to an evident recruitment of CAD-eYFP to the plasma membrane (top); $50 \mu \mathrm{M} 2$-APB failed to reverse the near plasma membrane CAD-eYFP localization in HEK293T cells (bottom). The right panels show profiles of fluorescence intensity of CAD-eYFP (green) and Orai1-mKate (red) along the lines imposed on the images. (b-e) Bar graphs show the change of PM fluorescence intensities of CAD-eYFP from various concentration 2-APB-treated experiments: $5 \mu \mathrm{M}$ 2-APB $(n=23), 25 \mu \mathrm{M} 2$-APB $(n=22), 50 \mu \mathrm{M} 2$-APB $(n=26)$, and $100 \mu \mathrm{M} 2$-APB $(n=26)$. (f) CAD-eYFP-induced constitutive Ca ${ }^{2+}$ entry in HEK293 cells co-expressing CAD-eYFP and Orail-eCFP was measured after $\mathrm{Ca}^{2+}$-free medium replaced with $1.8 \mathrm{mM}$ $\mathrm{Ca}^{2+}$ followed by $50 \mu \mathrm{M} 2$-APB addition. (g) Bar graph shows the Fura-2 ratiometric change after $50 \mu \mathrm{M} 2$-APB addition from 16 independent experiments shown in (f). (h) Change in FRET between Orai1-eCFP and CAD-eYFP in response to $50 \mu \mathrm{M} 2-\mathrm{APB}$ (bar).

concentrations of $2-\mathrm{APB}(50 \mu \mathrm{M})$ that is known to transiently potentiate and then inhibit CRAC currents resulted in a significant increase in CADOrai1 FRET [Fig. 6(h)].

\section{Discussion}

As the key subunit of CRACs, ER-resident STIM1 controls the opening of these channels. STIM1 is distributed throughout the ER in rest cells; depletion of $\mathrm{Ca}^{2+}$ store triggers the redistribution of STIM1 to sites of close apposition between the ER and the plasma membrane, which recruits Orai1 to STIM1 puncta, and the interaction of STIM1 and Orai1 can lead to the opening of CRACs. ${ }^{2,18,21,22}$
Therefore, STIM1 is essential for the recruitment of Orai1 to puncta and activation of SOCs, but the mechanisms about the formation of STIM1 puncta need further investigation.

The N-terminus of STIM1 consists of EF-hand and a sterile $\alpha$ motif (SAM); it locates in lumen of $\mathrm{ER}$ and is responsible for $\mathrm{Ca}^{2+}$ sensing. C-terminus of STIM1 is located in the cytosol, containing two coiled-coil regions overlapping with an ezrinradixin-moesin (ERM)-like domain, followed by a serine/proline and a lysine-rich region. ${ }^{2,35}$ Our experiments showed that STIM1 puncta were visible at the cell footprint by confocal microscope in HEK293 cells overexpressing STIM1 after depletion of $\mathrm{Ca}^{2+}$ stores, but for cells overexpressing Orai1 
alone, puncta could not be observed after $\mathrm{Ca}^{2+}$ depletion, and co-expression of STIM1 restored its ability to form puncta, which suggests that STIM1 can redistribute to ER-PM junctions in an Orai1independent manner, whereas recruitment of Orai1 to these sites depends on binding to STIM1. A STIM1 deletion mutant lacking the highly conserved lysine-rich region (STIM1- $\Delta \mathrm{K}$ ) has been reported to prevent puncta formation and SOC activation in some studies. ${ }^{6,19,21}$ Our research confirmed that depletion of lysine-rich region eliminated puncta formation upon $\mathrm{Ca}^{2+}$ store depletion when STIM1- $\Delta \mathrm{K}$ expressed alone in HEK293T cells. However, STIM1- $\Delta \mathrm{K}$ was recruited to ER-PM junctions to form puncta after store depletion while co-expressing with Orai1. Recently, three studies independently identified a crucial Orai-recruiting and activating domain within the ERM domain of STIM1, namely CRAC-activating domain CAD (CAD), ${ }^{21}$ STIM-Orai-activating region (SOAR), ${ }^{36}$ or Orai-activating small fragment (OASF). ${ }^{15}$ Our FRET experiment also probed the interaction between CAD and Orai1. Therefore, it was suggested that there might exist two mechanisms for STIM1 puncta formation. ${ }^{21}$ One is Orai1-independent pathway, which is mediated by the polybasic domain of STIM1. It is hypothesized that positively charged polybasic segment of STIM is recruited by negatively charged phospholipids such as PIP2 and PIP $3 .^{6}$ Another is the Orai1-dependent pathway, which is mediated by the direct interaction between Orai1 and CAD domain of STIM1.

2-APB was a reliable blocker of store-operated $\mathrm{Ca}^{2+}$ entry. ${ }^{29}$ Thus, we considered whether the inhibition of 2-APB on SOCE results from disruption of STIM1 puncta formation. Our results that 2-APB dose-dependently inhibited or reversed rearrangement of STIM1 in live cells overexpressing STIM1 alone are in line with previous studies, ${ }^{30,37}$ which suggested that 2-APB has the ability to affect the Orai1-independent mechanism of STIM1 puncta formation. It has been suggested that the C-terminal polybasic domain of STIM1 is an electrostatic phosphoinositide lipid-binding motif, which directs Orai1-independent formation of STIM1 puncta. ${ }^{2,6}$ We hypothesized that 2-APB inhibits or reverses rearrangement of STIM1 by disturbing the electrostatic attraction between STIM1 polybasic domain and PM phosphoinositides. To test this, we constructed plasmids of GFP$\mathrm{K}^{\text {STIM1 }}$ (contained the polybasic domains of

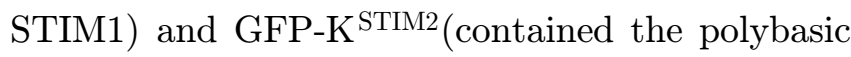
domains of STIM2) and monitored the intracellular localization of isolated STIM1/2 polybasic domain by confocal images in live cells. Experiments indicated that the eight charges in polybasic segment of a STIM1 monomer are too weak to induce PM binding on their own, and oligomerization of STIM1 may play an important role for binding to phosphoinositides at the PM. Our data also indicated that the binding of polybasic segment of STIM2 to $\mathrm{PM}$ was stronger than the binding of polybasic segment of STIM1 to PM. GFP-PLC $\delta$-PH and GFP-Rit tail, well-known polyphosphoinositide binding peptides, have been extensively used for in vitro biochemical studies of the interaction between polybasic amino acids and $\mathrm{PI}(4,5) \mathrm{P} 2$ and $\mathrm{PI}(3,4,5)$ P3, which resembles the polybasic tail of STIM protein. ${ }^{38}$ So we investigated the effect of 2 -APB on $\mathrm{PM}$ localization of GFP-K ${ }^{\mathrm{STIM}} 2$, GFP-PLC $\delta$-PH, and GFP-Rit tail to deduce the action mechanism of 2-APB to reverse Orai1-independent STIM1 puncta. We found that 2-APB could not disperse the PM localization of GFP-K ${ }^{\text {STIM2 }}$, GFP-Rit-tail, and GFP-PLC $\delta$-PH. Therefore, we concluded that 2-APB could not directly dissociate the electrostatic interaction between polybasic domain and PM phosphoinositides, but maybe enhance the intramolecular coupling between the CC1 and SOAR of STIM1 to inhibit or reverse rearrangement of STIM1 in the absence of Orai1 overexpression. ${ }^{39}$

In contrast, we found that 2-APB failed to inhibit the formation of puncta or disperse the puncta that had already been formed in HEK293 cells co-expressing STIM1/STIM1- $\Delta \mathrm{K}$ and Orai1 after depletion of $\mathrm{ER} \mathrm{Ca}^{2+}$ stores, which is in agreement with the recent report. ${ }^{37}$ Besides, 2-APB could not visibly alter near plasma membrane CAD-eYFP colocalization with Orai1. These results indicated that 2-APB had no effect on Orai1-dependent mechanism of STIM1 puncta formation. However, although 2-APB lost the ability to prevent or reverse STIM1 puncta, it still can completely inhibit store-operated $\mathrm{Ca}^{2+}$ entry in HEK293 cells coexpressing full-length STIM1 or STIM1- $\Delta \mathrm{K}$ with Orai1 and completely block CAD-YFP-induced constitutive $\mathrm{Ca}^{2+}$ entry. This discrepancy implies an additional inhibitory effect of 2-APB on SOCE downstream of STIM1, possibly on STIM1-Orai1 interaction or on the Orai1 channel itself. Interestingly, both high $(50 \mu \mathrm{M})$ and low $(5 \mu \mathrm{M})$ concentrations of 2-APB further enhanced the FRET 
between full-length STIM1 or STIM1-CT and Orai1, which suggest that effect of 2-APB may be to enhance interaction between STIM1 and Orai1. ${ }^{40,41}$ We also demonstrated that 2 -APB resulted in a pronounced increase in FRET between Orai1-CFP and CAD-YFP. The potentiating effect of low doses of 2-APB on CRACs may thus be explained by an increase in STIM1-Orai1 interaction. However, the increase in STIM1-Orai1 FRET and CAD-Orai1 FRET seen with high doses of 2-APB seems more difficult to explain. Therefore, the inhibitory effect of 2-APB on SOCE is unlikely to be caused by any ability to block STIM1 puncta formation or physically uncouple STIM1 and Orai1. Its inhibitory action might attribute to its ability to functionally uncouple STIM1 and Orai1 or direct inhibitory effects on Orai1 channel itself. Interestingly, recent study found that 2-APB can directly inhibit or enhance constitutive $\mathrm{Ca}^{2+}$ influx that is mediated by Orai1 mutants (Orai1-P245T, Orai1-V102A, and Orai1-V102C) ${ }^{39}$ Considering that high concentrations of 2-APB are known to transiently potentiate and then inhibit CRAC currents, we supposed that high doses of 2-APB might initially potentiate CRAC currents by increasing the STIM1 and Orai1 interaction, but soon combine with some specific parts of the opening Orai1 channels in lipid bilayer to completely block Orai1 channels followed by Orail channel activation and lead to inhibition of CRAC currents.

\section{Acknowledgments}

We thank Dr. Richard S. Lewis and Dr. Tamas Balla for cDNA constructs. This work was supported by the National Natural Sciences Foundation of China (Grant Nos. 31371217 and 30871311).

\section{References}

1. Y. Baba, T. Kurosaki, "Physiological function and molecular basis of STIM1-mediated calcium entry in immune cells," Immunol. Rev. 231(1), 174-188 (2009).

2. P. G. Hogan, R. S. Lewis, A. Rao, "Molecular basis of calcium signaling in lymphocytes: STIM and ORAI," Annu. Rev. Immunol. 28, 491-533 (2010).

3. A. B. Parekh, J. W. Putney Jr, "Store-operated calcium channels," Physiol. Rev. 85(2), 757-810 (2005).

4. J. W. Putney Jr , "A model for receptor-regulated calcium entry," Cell Calcium 7(1), 1-12 (1986).
5. J. T. Smyth, W. I. Dehaven, B. F. Jones, J. C. Mercer, M. Trebak, G. Vazquez, J. W. Putney Jr, "Emerging perspectives in store-operated $\mathrm{Ca}^{2+}$ entry: Roles of Orai, Stim and TRP," Biochim. Biophys. Acta 1763(11), 1147-1160 (2006).

6. J. Liou, M. Fivaz, T. Inoue, T. Meyer, "Live-cell imaging reveals sequential oligomerization and local plasma membrane targeting of stromal interaction molecule 1 after $\mathrm{Ca}^{2+}$ store depletion," Proc. Natl Acad. Sci. USA 104(22), 9301-9306 (2007).

7. J. Liou, M. L. Kim, W. D. Heo, J. T. Jones, J. W. Myers, J. E. Ferrell Jr, T. Meyer, "STIM is a $\mathrm{Ca}^{2+}$ sensor essential for $\mathrm{Ca}^{2+}$-store-depletion-triggered $\mathrm{Ca}^{2+}$ influx," Curr. Biol. 15(13), 1235-1241 (2005).

8. R. M. Luik, B. Wang, M. Prakriya, M. M. Wu, R. S. Lewis, "Oligomerization of STIM1 couples ER calcium depletion to CRAC channel activation," $\mathrm{Na}$ ture 454(7203), 538-542 (2008).

9. R. M. Luik, M. M. Wu, J. Buchanan, R. S. Lewis, "The elementary unit of store-operated $\mathrm{Ca}^{2+}$ entry: Local activation of CRAC channels by STIM1 at ER-plasma membrane junctions," J. Cell Biol. 174(6), 815-825 (2006).

10. J. Roos, P. J. DiGregorio, A. V. Yeromin, K. Ohlsen, M. Lioudyno, S. Zhang, O. Safrina, J. A. Kozak, S. L. Wagner, M. D. Cahalan, G. Velicelebi, K. A. Stauderman, "STIM1, an essential and conserved component of store-operated $\mathrm{Ca}^{2+}$ channel function," J. Cell Biol. 169(3), 435-445 (2005).

11. S. L. Zhang, Y. Yu, J. Roos, J. A. Kozak, T. J. Deerinck, M. H. Ellisman, K. A. Stauderman, M. D. Cahalan, "STIM1 is a $\mathrm{Ca}^{2+}$ sensor that activates CRAC channels and migrates from the $\mathrm{Ca}^{2+}$ store to the plasma membrane," Nature 437(7060), 902905 (2005).

12. M. Prakriya, S. Feske, Y. Gwack, S. Srikanth, A. Rao, P. G. Hogan, "Orai1 is an essential pore subunit of the CRAC channel," Nature 443(7108), 230-233 (2006).

13. M. Vig, W. I. DeHaven, G. S. Bird, J. M. Billingsley, H. Wang, P. E. Rao, A. B. Hutchings, M. H. Jouvin, J. W. Putney, J. P. Kinet, "Defective mast cell effector functions in mice lacking the CRACM1 pore subunit of store-operated calcium release-activated calcium channels," Nat. Immunol. 9(1), 89-96 (2008).

14. A. V. Yeromin, S. L. Zhang, W. Jiang, Y. Yu, O. Safrina, M. D. Cahalan, "Molecular identification of the CRAC channel by altered ion selectivity in a mutant of Orai," Nature 443(7108), 226-229 (2006).

15. M. Muik, M. Fahrner, I. Derler, R. Schindl, J. Bergsmann, I. Frischauf, K. Groschner, C. Romanin, "A cytosolic homomerization and a modulatory domain within STIM1 C terminus determine coupling to ORAI1 channels," J. Biol. Chem. 284(13), 8421-8426 (2009). 
16. P. B. Stathopulos, G. Y. Li, M. J. Plevin, J. B. Ames, M. Ikura, "Stored $\mathrm{Ca}^{2+}$ depletion-induced oligomerization of stromal interaction molecule 1 (STIM1) via the EF-SAM region: An initiation mechanism for capacitive $\mathrm{Ca}^{2+}$ entry," J. Biol. Chem. 281(47), 35855-35862 (2006).

17. M. M. Wu, J. Buchanan, R. M. Luik, R. S. Lewis, " $\mathrm{Ca}^{2+}$ store depletion causes STIM1 to accumulate in ER regions closely associated with the plasma membrane," J. Cell Biol. 174(6), 803-813 (2006).

18. P. Xu, J. Lu, Z. Li, X. Yu, L. Chen, T. Xu, "Aggregation of STIM1 underneath the plasma membrane induces clustering of Orai1," Biochem. Biophys. Res. Commun. 350(4), 969-976 (2006).

19. G. N. Huang, W. Zeng, J. Y. Kim, J. P. Yuan, L. Han, S. Muallem, P. F. Worley, "STIM1 carboxylterminus activates native SOC, I(crac) and TRPC1 channels," Nat. Cell Biol. 8(9), 1003-1010 (2006).

20. Z. Li, J. Lu, P. Xu, X. Xie, L. Chen, T. Xu, "Mapping the interacting domains of STIM1 and Orai1 in $\mathrm{Ca}^{2+}$ release-activated $\mathrm{Ca}^{2+}$ channel activation," J. Biol. Chem. 282(40), 29448-29456 (2007).

21. C. Y. Park, P. J. Hoover, F. M. Mullins, P. Bachhawat, E. D. Covington, S. Raunser, T. Walz, K. C. Garcia, R. E. Dolmetsch, R. S. Lewis, "STIM1 clusters and activates CRAC channels via direct binding of a cytosolic domain to Orai1," Cell 136(5), 876-890 (2009).

22. J. Soboloff, B. S. Rothberg, M. Madesh, D. L. Gill, "STIM proteins: Dynamic calcium signal transducers," Nat. Rev. Mol. Cell Biol. 13(9), 549-565 (2012).

23. S. Feske, M. Prakriya, "Conformational dynamics of STIM1 activation," Nat. Struct. Mol. Biol. 20(8), 918-919 (2013).

24. Y. Zhou, P. Srinivasan, S. Razavi, S. Seymour, P. Meraner, A. Gudlur, P. B. Stathopulos, M. Ikura, A. Rao, P. G. Hogan, "Initial activation of STIM1, the regulator of store-operated calcium entry," Nat. Struct. Mol. Biol. 20(8), 973-981 (2013).

25. S. Srikanth, H. J. Jung, K. D. Kim, P. Souda, J. Whitelegge, Y. Gwack, "A novel EF-hand protein, CRACR2A, is a cytosolic $\mathrm{Ca}^{2+}$ sensor that stabilizes CRAC channels in T cells," Nat. Cell Biol. 12(5), 436-446 (2010).

26. R. Palty, A. Raveh, I. Kaminsky, R. Meller, E. Reuveny, "SARAF inactivates the store operated calcium entry machinery to prevent excess calcium refilling," Cell 149(2), 425-438 (2012).

27. S. Srikanth, M. Jew, K. D. Kim, M. K. Yee, J. Abramson, Y. Gwack, "Junctate is a $\mathrm{Ca}^{2+}$-sensing structural component of Orail and stromal interaction molecule 1 (STIM1)," Proc. Natl Acad. Sci. USA 109(22), 8682-8687 (2012).
28. T. Maruyama, T. Kanaji, S. Nakade, T. Kanno, K. Mikoshiba, "2APB, 2-aminoethoxydiphenyl borate, a membrane-penetrable modulator of Ins (1,4,5)P3-induced $\mathrm{Ca}^{2+}$ release," J. Biochem. 122 (3), 498-505 (1997).

29. M. D. Bootman, T. J. Collins, L. Mackenzie, H. L. Roderick, M. J. Berridge, C. M. Peppiatt, "2-aminoethoxydiphenyl borate (2-APB) is a reliable blocker of store-operated $\mathrm{Ca}^{2+}$ entry but an inconsistent inhibitor of InsP3-induced $\mathrm{Ca}^{2+}$ release," FASEB J. 16(10), 1145-1150 (2002).

30. W. I. DeHaven, J. T. Smyth, R. R. Boyles, G. S. Bird, J. W. Putney Jr, "Complex actions of 2-aminoethyldiphenyl borate on store-operated calcium entry," J. Biol. Chem. 283(28), 19265-19273 (2008).

31. H. T. Ma, K. Venkatachalam, J. B. Parys, D. L. Gill, "Modification of store-operated channel coupling and inositol trisphosphate receptor function by 2-aminoethoxydiphenyl borate in DT40 lymphocytes," J. Biol. Chem. 277(9), 6915-6922 (2002).

32. M. Prakriya, R. S. Lewis, "Potentiation and inhibition of $\mathrm{Ca}^{2+}$ release-activated $\mathrm{Ca}^{2+}$ channels by 2 -aminoethyldiphenyl borate (2-APB) occurs independently of IP3 receptors," J. Physiol. 536(Pt 1), 3-19 (2001).

33. C. Peinelt, A. Lis, A. Beck, A. Fleig, R. Penner, "2APB directly facilitates and indirectly inhibits STIM1-dependent gating of CRAC channels," J. Physiol. 586(13), 3061-3073 (2008).

34. N. Scrimgeour, T. Litjens, L. Ma, G. J. Barritt, G. Y. Rychkov, "Properties of Orai1 mediated storeoperated current depend on the expression levels of STIM1 and Orai1 proteins," J. Physiol. 587(Pt 12), 2903-2918 (2009).

35. R. T. Williams, P. V. Senior, L. Van Stekelenburg, J. E. Layton, P. J. Smith, M. A. Dziadek, "Stromal interaction molecule 1 (STIM1), a transmembrane protein with growth suppressor activity, contains an extracellular SAM domain modified by N-linked glycosylation," Biochim. Biophys. Acta 1596(1), 131-137 (2002).

36. J. P. Yuan, W. Zeng, M. R. Dorwart, Y. J. Choi, P. F. Worley, S. Muallem, "SOAR and the polybasic STIM1 domains gate and regulate Orai channels," Nat. Cell Biol. 11(3), 337-343 (2009).

37. B. Zeng, G. L. Chen, S. Z. Xu, "Store-independent pathways for cytosolic STIM1 clustering in the regulation of store-operated $\mathrm{Ca}(2+)$ influx," Biochem. Pharmacol. 84(8), 1024-1035 (2012).

38. W. D. Heo, T. Inoue, W. S. Park, M. L. Kim, B. O. Park, T. J. Wandless, T. Meyer, "PI(3,4,5)P3 and $\mathrm{PI}(4,5) \mathrm{P} 2$ lipids target proteins with polybasic clusters to the plasma membrane," Science 314(5804), 1458-1461 (2006). 
39. M. Wei, Y. Zhou, A. Sun, G. Ma, L. He, L. Zhou, S. Zhang, J. Liu, S. L. Zhang, D. L. Gill, Y. Wang, "Molecular mechanisms underlying inhibition of STIM1-Orail-mediated $\mathrm{Ca}^{2+}$ entry induced by 2-aminoethoxydiphenyl borate," Pflugers Archiv. Eur. J. Physiol. 468(11-12), 2061-2074 (2016).

40. L. Navarro-Borelly, A. Somasundaram, M. Yamashita, D. Ren, R. J. Miller, M. Prakriya,
"STIM1-Orai1 interactions and Orai1 conformational changes revealed by live-cell FRET microscopy," J. Physiol. 586(Pt 22), 5383-5401 (2008).

41. Y. Wang, X. Deng, Y. Zhou, E. Hendron, S. Mancarella, M. F. Ritchie, X. D. Tang, Y. Baba, T. Kurosaki, Y. Mori, J. Soboloff, D. L. Gill, "STIM protein coupling in the activation of Orai channels," Proc. Natl Acad. Sci. USA 106(18), 7391-7396 (2009). 